\title{
LONG-TERM EFFECTS OF MORE PUNITIVE LEGISLATION CONCERNING THE USE OF MOBILE PHONE DURING DRIVING IN PORTUGAL
}

\author{
Mário B. Godinho \\ Faculdade de Motricidade Humana, \\ Technical University of Lisbon \\ Portugal \\ E-mail: mgodinho@fmh.utl.pt
}

\begin{abstract}
Summary: The purpose of this study was to determine whether a change in legislation regarding the use of handheld cell phones in Portugal provoked a substantial reduction of their use and if one year latter this reduction was still visible. Drivers' handheld cell phone use was observed prior to the implementation of more punitive legislation, immediately after, 6 months and one year after the change in legislation. Estimated age ( + or -40 years old) and gender was also registered for cellular telephones users. The counts were taken over 20 one-hour occasions equally divided over the four periods of observation and they were performed at the IC19 near Queluz, which is one of the roads with the most intense traffic flows of the region, for a total of 66841 observations. Results showed a considerable reduction from $1.29 \%$ of drivers before the law to $0.67 \%$ immediately after. However, one year later, the results $(1.27 \%)$ were analogous to the initial observations. Phone users were predominantly male $(75.35 \%)$, but this percentage is equivalent to all road users, so a gender trend was not found. On the contrary, we found a higher trend of younger drivers using mobile phones while driving. One of the most probable reasons for the main effect found was that as time goes by, drivers realize the low risk of being charged and punished for phone use while driving. The more punitive measures implemented with the new law regarding phone use showed no long-term effects.
\end{abstract}

\section{INTRODUCTION}

Distraction is considered one of the important factors in automobile accidents. The rapid proliferation of portable cellular telephones, and their use while driving, may degrade human driving performance considerably. Many drivers sincerely believe that they have the talent to use the mobile phone simultaneously with driving. There is some evidence that the use of handheld phones quadruples the risk of a collision during the brief period of a call (Redelmeier \& Tibshirani, 1997). Other studies found a noticeably higher risk (Violanti, 1997) and distinguished male from females, with significantly higher risk for males.

Even a hands-free mobile phone conversation can compromise the driving task. Petersen et al. (2003) reported that speaking on a hands-free mobile phone while driving affected critical components of the perception-action cycle. Reaction time can also increase (by about $80 \mathrm{~ms}$ ) when a conversation takes place with a handheld or hands free mobile phone (Consiglio, et al., 2003). In a recent meta-analysis performed by Horrey and Wickens (2006), the impact of a phone conversation on reaction time was calculated to be around $130 \mathrm{~ms}$, with clear results in 
terms of performance costs. To cope with the additional stress imposed by a phone conversation (regardless of the type of phone used), subjects reveal higher workloads or voluntarily reduce performance goals (Rakauskas, et al., 2004).

The degree of the negative effects of portable phone use while driving is dependent on the complexity of the conversation and of the driving situation. The more difficult and complex the conversation, the more dangerous the effect on driving performance.

There has been very little research done, and none in Portugal, on actual exposure rates on the road whilst using a phone. The precise effects of using mobile phones while driving in Portugal are not known. However, because of the possible increase in risks, there was a legislative effort to try to restrict cell phone use on the road, with an increased fine for drivers using hand held phones while driving. The law does not apply to hands-free devices. This change in legislation took place on the 26 of March 2005. Media coverage was considerable in the proximity of the date on which the law took full effect. Six month and one year after, there were no visible campaigns in the media about this issue.

The introduction of restrictive legislation was found to have a strong effect on the use of handheld cell phones (McCartt, et al., 2003). However, one year after, that effect was found to dissipate (McCartt \& Geary, 2004).

The aim of this research was to evaluate driver behavior before, immediately after, 6 months and one year after the implementation of the more punitive legislation concerning the use of mobile phones during driving.

\section{METHODS}

\section{Procedures}

The data were collected by a roadside observer. Five one-hour training sessions were performed by two observers to guarantee the correctness of the data gathering. As the training evolved, consistency between observers increased. At the end of the training period, a $98 \%$ agreement rate was obtained. In a period of approximately 1 hour, 3351 observations were made by both observers and the final count was 47 and 48 for handheld mobile phone users.

The procedure was similar to the one used by Horberry, et al. (2001). Observers used a Manutan handheld counter, which counts the number of all motor vehicles (except motorbikes) traveling past. A tape recorder was used to register the drivers who were using a handheld mobile phone. Age and gender were also registered. Due to difficulties in age estimation, the drivers were classified as over or under 40 years old. The counts were taken over a period of 60 minutes, between 15:00 and 17:30 hours, during weekdays from January 2005 through April 2006. Observations were made that the weather was fine, dry and bright.

An observation of the drivers' characteristics was performed in 8 periods of 10 minutes scattered along the 4 observation moments and at exactly the same observation spot. These periods often 
followed the observation of phone users. During these periods, gender and age rates were examined.

The first data collection session took place before (Bf) the implementation of the more punitive legislation (from January 2005 until March 2005). The second was in April 2005, immediately after (IA) the change in the above-mentioned law. Repeated measurements were made 6 months (6M) later in September 2005, and one year (1Y) later in April 2006. All observations were made under good visibility conditions.

The location for all observations was the IC19, a three-lane road near Queluz, and always in the same direction of traffic flow (Lisbon-Sintra) to allow for better visibility of the interior of vehicles. The reason for choosing this location was that it has one of the most intense traffic flows in the neighborhood of the metropolitan area of Lisbon.

All motor vehicles, except motorbikes, in all three lanes were screened. The use of mobile phone was counted only when there was a clear view of the vehicle and the phone itself. A vehicle that was not screened, either due to difficulty in judging the use or not of mobile phone or for some other observation difficulty, was not considered.

\section{RESULTS}

A total of 66841 observations were made during all periods $(\mathrm{Bf}=15746, \mathrm{IA}=16851,6 \mathrm{M}=$ 18567 and $1 \mathrm{Y}=15677)$.

Data analysis provided an overall, sex and age rates of mobile phone use. Rates are reported as number of mobile phone users/100 observations. Table 1 presents overall data for the four periods of observation.

Table 1. Minimum, maximum, mean and standard deviation for before (Bf), immediately after (IA), 6 months (6M) and 1 year after (1Y) the implementation of a more punitive law

\begin{tabular}{|l|c|c|c|c|c|}
\hline & $\mathrm{N}$ & Min. & Max. & Mean & Std. Dev. \\
\hline Bf & 5 & 1.100 & 1.400 & 1.288 & 0.112 \\
\hline IA & 5 & 0.540 & 0.780 & 0.674 & 0.101 \\
\hline $6 \mathrm{M}$ & 5 & 0.900 & 1.200 & 1.094 & 0.117 \\
\hline 1Y & 5 & 1.100 & 1.500 & 1.270 & 0.165 \\
\hline
\end{tabular}

In Figure 1 it is possible to observe a considerable reduction of occurrences immediately after the implementation of the more punitive law regarding mobile phone use while driving. This reduction had dissipated one year after reaching the levels obtained in the Before period. A Chi Square analysis showed no significant differences between the periods of observation (Chi Square $=0.202, \mathrm{p}>0.05)$. 


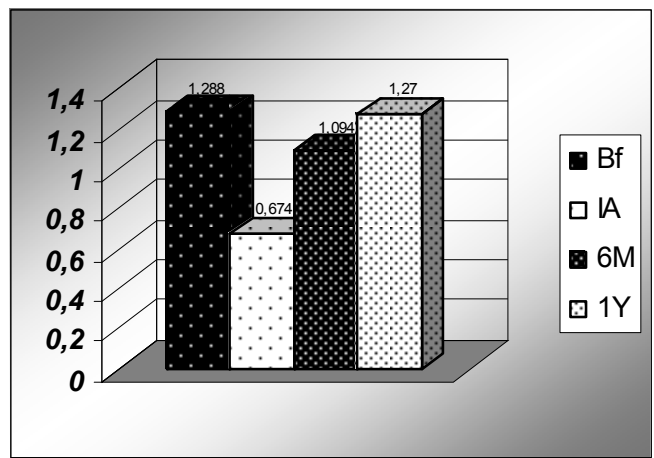

Figure 1. Mean values for before (Bf), immediately after (IA), 6 months (6M) and 1 year after (1Y) the implementation of a more punitive law

The relative frequency of male/female and over/under 40 years old is evident in Table 2. It is obviously a relatively stable percentage (particularly the male/female frequency) among the four periods of observation.

Table 2. Relative percentage of Males (M) and Females (F), -40 and +40 years old drivers using mobile phones while driving in the 4 observation periods

\begin{tabular}{|c|c|r|r|r|}
\hline & M & \multicolumn{1}{c|}{ F } & \multicolumn{1}{c|}{40} & \multicolumn{1}{c|}{+40} \\
\hline Bf & 74.8 & 25.2 & 66.4 & 33.6 \\
\hline IA & 77.8 & 22.2 & 54.4 & 45.6 \\
\hline 6M & 76.6 & 23.4 & 65.8 & 34.2 \\
\hline 1Y & 72.2 & 27.8 & 66.4 & 33.6 \\
Total & 75.35 & 24.65 & 63.25 & 36.75 \\
\hline
\end{tabular}

In 4 different periods (10 minutes each) we observed every car that passed by the observer solely in terms of the sex of the driver. The percentage found was $74.5 \%$ men and $25.5 \%$ women. This relation is very similar to the one observed with drivers using a mobile phone, which means that there is no gender trend in what concerns the use of this object while driving.

The same procedure was followed for age. The percentage found was $56.4 \%$ of -40 years old and $43.6 \%$ of +40 years old drivers. If we consider the overall data (cf. Table 2 ), it is evident that younger drivers use more mobile phones while driving than older drivers.

\section{DISCUSSION}

Immediately after the change in the law there was a significant decrease (although not statistically) in the use of mobile phones while driving. Approximately half the number of drivers screened in the Bf period were registered after the change in the punitive measures. It is evident that this measure had the intended result. However, one year later, the use rate had increased considerably, to levels identical to the pre-change-law period. This trend was identical to what was found in the McCartt and Geary (2004) study, in which 16 months after the ban, the levels of mobile phone use were identical to the pre-law period. The reason for this may be the 
fact that, as time goes by, drivers realize the low risk of being charged and punished for phone use while driving.

It was found that phone users were predominantly male $(75.35 \%)$, which is in agreement with the observations of Horberry, et al. (2001), in which the authors refer to a predominance of 78\% male users. Similar data (77.9\%) was found in the research of Townsend (2006) in a context (New Zealand) in which there is no restrictive legislation regarding the use of mobile phones while driving. The relation of male/female drivers in all drivers in our study is identical to the mobile phone users, so a gender trend on phone use while driving was not observed.

With reference to age, we found that $63.25 \%$ of the drivers who were screened as using a mobile phone were under 40 years old. This percentage is very similar to the one found in the Horberry, et al. study (64\%). However, a record of $56.4 \%$ of under- 40 was found for all drivers, independently of using or not using a mobile phone. This indicates a larger rate of use of cellular phones in vehicles by younger drivers. This trend may be associated with the felt difficulties of older drivers.

Our study has some limitations. Observation was limited to 5 different hours in each of the 4 periods, which may not be representative of the true rates of mobile phone use. Some vehicles were missed because of registering procedures and others for inability to observe (reflections or position of hands covering potentially the mobile phone), so the number recorded is probably an underestimate.

Further observations are recommended to identify possible trends in handheld mobile phone usage while driving. A search for possible geographical-area and time-of-day effects is advisable. Possible relations with the mobile phone market may be also interesting. In spite of having some congruence with other studies (e.g., Horberry, et al., 2001) a generalization of these results to other areas of Portugal would surely be problematic.

Punitive measures per se showed limitations in terms of long-term effects. A combination of legal measure and educational campaigns should be advisable. Considering the harmful evidence of pursuing a conversation while driving, an enlargement of the scope of the educational measures should consider the limitations of using a hands-free device.

The long-term limitations of these punitive measures emphasize the need for more continued and consistent action.

\section{REFERENCES}

Consiglio, W., Driscoll, P., Witte, M., \& Berg, W. P. (2003). Effect of cellular telephone conversations and other potential interference on reaction time in a braking response. Accident Analysis and Prevention, 35, 495-500.

Horberry, T., Bubnich, C., Hartley, L., \& Lamble, D. (2001). Drivers' use of hand-held mobile phones in western Australia. Transportation Research, Part F: Traffic Psychological and Behaviour, 4, 213-218. 
Horrey, W.J. \& Wickens, C.D. (2006). Examining the impact of cell phone conversations on driving using meta-analytic techniques. Human Factors, 48 (1), 196-205.

McCartt, A.T., Braver, E.R., \& Geary, L.L. (2003). Drivers' use of handheld cell phones before and after New York State's cell phone law. Preventive Medicine, 36 (5), 629-635.

McCartt, A.T., \& Geary, L.L. (2004). Longer term effects of New York State's law on drivers' handheld cell phone use. Injury Prevention, 10, 11-15.

Petersen, A., Treffner, P., \& Barrett, R. (2003). Mobile phones and driving: Affordances and attention. In S. Rogers \& J. Effken (Eds.), Studies in perception and action VII. Mahwah, N.J.: Erlbaum, 140-144.

Rakauskas, M., Gugerty, L., \& Ward, N.J. (2004). Effects of cell phone conversations on driving performance with naturalistic conversations. Journal of Safety Research, 35, 435-464.

Redelmeier, D.A., \& Tibshirani, R.J. (1997). Association between cellular telephone calls and motor vehicle collisions. New England Journal of Medicine, 336 (7), 453-458.

Towsend, M. (2006). Motorists' use of hand held cell phones in New Zealand: An observational study. Accident Analysis and Prevention, 38, 748-750.

Violanti, J.M. (1997). Cellular phones and traffic accidents. Public Health, 111, 423-428. 